ARTICLE OPEN

\title{
Experimental critical quantum metrology with the Heisenberg scaling
}

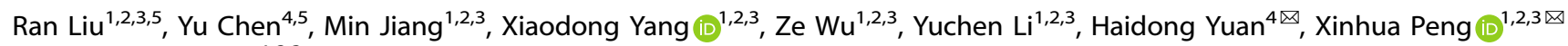
and Jiangfeng Du (iD) ${ }^{1,2,3}$

Critical quantum metrology, which exploits quantum critical systems as probes to estimate a physical parameter, has gained increasing attention recently. However, the critical quantum metrology with a continuous quantum phase transition (QPT) is experimentally challenging since a continuous QPT only occurs at the thermodynamic limit. Here, we propose an adiabatic scheme on a perturbed Ising spin model with a first-order QPT. By introducing a small transverse magnetic field, we can not only encode an unknown parameter in the ground state but also tune the energy gap to control the evolution time of the adiabatic passage. Moreover, we experimentally implement the critical quantum metrology scheme using nuclear magnetic resonance techniques and show that at the critical point the precision achieves the Heisenberg scaling as $1 / T$. As a theoretical proposal and experimental implementation of the adiabatic scheme of critical quantum metrology and its advantages of easy implementation, inherent robustness against decays and tunable energy gap, our adiabatic scheme is promising for exploring potential applications of critical quantum metrology on various physical systems.

npj Quantum Information (2021)7:170; https://doi.org/10.1038/s41534-021-00507-x

\section{INTRODUCTION}

Quantum metrology, which makes use of the superposition and entanglement, can achieve far better precision than the classical schemes $^{1-3}$. In the conventional scheme of quantum metrology, the estimation of an unknown parameter is typically achieved by first preparing a probe state, then letting the probe evolve under a dynamics that encodes the unknown parameter, the value of the parameter can then be estimated from the evolved state via a suitable measurement ${ }^{1-4}$.

With an entangled probe state, quantum metrology can potentially enhance the precision from the classical shot noise limit, which scales as $N^{-1 / 2}$, to the Heisenberg limit, which scales as $N^{-1}$, here $N$ is the number of the probes ${ }^{1,2,4-13}$. The classical shot noise limit and the Heisenberg limit can also be considered in terms of the evolution time, $T$, where the precision scales as $T^{-1 / 2}$ for the shot noise limit and $T^{-1}$ for the Heisenberg limit ${ }^{14,15}$. For the conventional scheme, which consists of preparation, evolution and measurement, the ability to prepare highly entangled probe states or maintain a sufficiently long coherent evolution is essential to achieve a precision beyond the classical limit. This quantum advantage is not achievable in general for systems subject to noise.

Recently, the critical quantum metrology ${ }^{16-28}$ has attracted increasing theoretical interest since it combines the advantages of the intrinsic robustness due to the adiabatic evolution ${ }^{16,29}$ and high sensitivity near the critical point. Similar to the adiabatic quantum computation ${ }^{30-32}$, the critical quantum metrology with adiabatic evolution starts with the ground state of an initial Hamiltonian, which is easy to prepare, then evolves adiabatically to the ground state of the final Hamiltonian close to the critical point that encodes the unknown parameter. However, previous protocols typically consider systems with a continuous quantum phase transition (QPT), which only exists at the thermal dynamical limit, and the minimal energy gap at the critical point is also in general fixed which limits the speed of the adiabatic evolution. Such requirements impose great challenges on the experimental realization of the critical quantum metrology.

In this work, we overcome these challenges and propose an adiabatic scheme by employing a perturbed two-spin system with a first-order QPT where the energy gap can be tuned by introducing a small transverse magnetic field which lifts the energy crossing and controls the time required by the adiabatic passage. This can also be used to tune the trade-off between the precision and the bandwidth of the estimation. Moreover, we experimentally implement the scheme using a two-spin nuclear magnetic resonance (NMR) system and demonstrate a precision at the Heisenberg scaling of the probe time $T$ as $1 / T$. The adiabatic scheme is inherent robust against the decay since it remains at the ground state during the evolution, which we also verify with numerical simulations. As a first theoretical proposal and experimental implementation of the adiabatic scheme of critical quantum metrology, it opens an avenue for exploring potential applications of critical quantum metrology on various physical systems.

\section{RESULTS}

\section{Quantum critical model}

The quantum critical model employed in our adiabatic scheme of the critical quantum metrology is a two-spin-1/2 Ising model with the Hamiltonian

$$
\mathscr{H}_{\text {Ising }}=B_{z}\left(\sigma_{z}^{1}+\sigma_{z}^{2}\right)+\sigma_{z}^{1} \sigma_{z}^{2}
$$

where $\sigma_{z}^{i}$ is Pauli operator on the ith spin, $B_{z}$ is a longitudinal magnetic field to be estimated. The ground state of the

${ }^{1}$ Hefei National Laboratory for Physical Sciences at the Microscale and Department of Modern Physics, University of Science and Technology of China, Hefei 230026, China. ${ }^{2}$ CAS Key Laboratory of Microscale Magnetic Resonance, University of Science and Technology of China, Hefei 230026, China. ${ }^{3}$ Synergetic Innovation Center of Quantum Information and Quantum Physics, University of Science and Technology of China, Hefei 230026, China. ${ }^{4}$ Department of Mechanical and Automation Engineering, The Chinese University of Hong Kong, Shatin, Hong Kong SAR, China. ${ }^{5}$ These authors contributed equally: Ran Liu, Yu Chen. ${ }^{凶}$ email: hdyuan@mae.cuhk.edu.hk; xhpeng@ustc.edu.cn 
Hamiltonian is given by

$$
\left|g\left(B_{z}\right)\right\rangle=\left\{\begin{array}{cc}
|00\rangle ; & B_{z} \leq-1, \\
\frac{|01\rangle \pm|10\rangle}{\sqrt{2}} ; & -1 \leq B_{z} \leq 1, \\
|11\rangle ; & B_{z} \geq 1,
\end{array}\right.
$$

with the corresponding eigenenergies $1+2 B_{z \prime}-1$ and $1-2 B_{z \prime}$ respectively. At $B_{z}= \pm 1$, a first-order QPT occurs, where the energy-level crossing exactly exists as well as a sudden change of its ground state. The ground state has a degeneracy of 2 when $-1<B_{z}<1$. The degeneracy, however, can be lifted by restricting to the symmetric triplet space ${ }^{33}$. Intuitively as the Hamiltonian is invariant under the exchange of the two spins, if the initial state is symmetric then the state will remain in the symmetric space during the evolution. We can thus only consider the symmetric states and the adiabatic evolution is only constrained by the energy gap of the effective Hamiltonian on the symmetric space ${ }^{34}$.

The ground state on the symmetric space, however, still does not provide a precise information of $B_{z}$. To enable the estimation of $B_{z}$, we need a one-to-one correspondence between $B_{z}$ and the ground state. To achieve that we can add a small transverse field with $B_{x} \ll 1$,

$$
\widetilde{\mathscr{H}}_{\text {Ising }}=B_{z}\left(\sigma_{z}^{1}+\sigma_{z}^{2}\right)+B_{x}\left(\sigma_{x}^{1}+\sigma_{x}^{2}\right)+\sigma_{z}^{1} \sigma_{z}^{2},
$$

which preserves the symmetry. The energy-level crossing is lifted and the energy gap opens linearly with $B_{x}$ at the critical points $B_{z}= \pm 1^{35}$. The transverse field thus transforms the singular jump at the critical point to a non-singular transition over a finite width. By tuning $B_{x}$, we can adjust the width and the rate of change near the critical point. This transverse field can also be used to tune the energy gap, which determines the evolution time of the adiabatic passage. Our adiabatic scheme of critical quantum metrology for measuring a magnetic field mainly exploits these properties of the first-order QPT of the Ising model near its critical point $B_{z}=1$.

As a proof of principle, we focus on the local estimation where $B_{z}$ is within a small neighborhood of a known value. The precision of the local estimation can be characterized by the quantum Cramer-Rao bound (QCRB) ${ }^{1-3,36,37}$ as

$$
\delta B_{z}^{2} \geq \frac{1}{v F_{Q}}
$$

here $v$ is the number of repetitions of the experiment and $F_{\mathrm{Q}}$ is the quantum Fisher information $(\mathrm{QFI})^{1-3}$ of the final state, $|\widetilde{g}\rangle$. The ground states of $\widetilde{\mathscr{H}}_{\text {Ising }}$ are very close to those of $\mathscr{H}_{\text {Ising, }}$ except in the vicinity of the critical points, where the transverse field mixes them, thus avoiding the energy-level crossing. In this region, it is sufficient to consider the two lowest energy state $\left\{|a\rangle:=|11\rangle,|b\rangle:=\frac{|01\rangle+|10\rangle}{\sqrt{2}}\right\}$. The effective Hamiltonian on the two lowest energy levels can be written as

$$
\mathscr{H}_{\text {eff }}=-\left|B_{z}\right| \mathbf{1}_{2}+\left(1-\left|B_{z}\right|\right) \sigma_{z}+\sqrt{2} B_{x} \sigma_{x}
$$

where $\mathbf{1}_{n}$ denotes the $n \times n$ identity operator. When $B_{z}>0$, the ground state of the effective Hamiltonian can be written as

$$
\left|\widetilde{g}\left(B_{z}\right)\right\rangle=-\sin \frac{\theta}{2}|a\rangle+\cos \frac{\theta}{2}|b\rangle,
$$

where $\tan \theta=\frac{\sqrt{2} B_{x} 38}{1-B_{z}}$. The QFI of the ground state

$$
F_{\mathrm{Q}}(|\widetilde{g}\rangle)=4\left(\left\langle\partial_{B_{z}} \widetilde{g} \mid \partial_{B_{z}} \widetilde{g}\right\rangle-\left|\left\langle\widetilde{g} \mid \partial_{B_{z}} \widetilde{g}\right\rangle\right|^{2}\right)
$$

can then be obtained as

$$
F_{\mathrm{Q}}(|\widetilde{g}\rangle)=\frac{2 B_{x}^{2}}{\left[\left(1-B_{z}\right)^{2}+2 B_{x}^{2}\right]^{2}} .
$$

Near the critical point $B_{z}=1, F_{\mathrm{Q}}(|\widetilde{g}\rangle) \approx \frac{1}{2 B_{x}^{2}}$, which suggests an arbitrarily high precision when $B_{x} \rightarrow 0$. However, the closing of the energy gap when $B_{x} \rightarrow 0$ implies a critical slowing down and an inevitable growth of the protocol duration. A small, finite $B_{x}$ reconciles this contradiction, as well as enables the adiabatic preparation of the ground state at the critical point $B_{z}=1$. In the following, we shall show that critical quantum probes can achieve a Heisenberg scaling of the sensitivity via a suitable local design of the adiabatic passage to the ground state approaching the critical point.

\section{Experimental protocol}

We can implement the adiabatic evolution with an additional control field along the $z$-direction as

$$
\widetilde{\mathscr{H}}(t)=\left[B_{z}+B_{C}(t)\right]\left(\sigma_{z}^{1}+\sigma_{z}^{2}\right)+B_{x}\left(\sigma_{x}^{1}+\sigma_{x}^{2}\right)+\sigma_{z}^{1} \sigma_{z}^{2},
$$

where $B_{c}(t)$ is the control field which adiabatically changes from a large value to zero. This preserves the symmetry of the evolution. In the experiment, $B_{z}$ and $B_{c}(t)$ is combined as a single field which is changed from a large value to some $B_{z}$, whose value is then estimated by proper measurements on the final state. The calibration of the measured effective magnetic field can be found in Supplementary Note 6 . To gauge the practical advantage near the critical point, however, we also need to evaluate the cost, which is the time, $T$, required for the adiabatic evolution. A QFI scaling as $T^{2}$ corresponds to the Heisenberg scaling ${ }^{14,15,39}$, while a QFI scaling as $T$ corresponds the shot noise limit.

We consider the time required by the adiabatic evolution from an initial large $B_{z 0}$ to the critical point, $B_{z c} \approx 1$. For the local precision limit where the field is within a small neighborhood of a known field, if the field to be estimated is not near the critical point, we can shift it by compensating it with an additional known field. For general unknown field that is not within a small neighborhood, this can be achieved through the two-step adaptive method ${ }^{40,41}$. In this two-step method, the experiment is repeated where the first few experiments are used to obtain a rough estimation of the unknown field, with this rough estimation the field can then be shifted to near the critical point in the following experiments. The detailed procedure can be seen in Supplementary Note 2.

The adiabatic path can be described as

$$
\mathscr{H}_{\text {ad }}[A(s)]=[1-A(s)] \widetilde{\mathscr{H}}_{\text {lsing }}\left(B_{z 0}\right)+A(s) \widetilde{\mathscr{H}}_{\text {lsing }}\left(B_{z c}\right),
$$

where $\widetilde{\mathscr{H}}_{\text {Ising }}\left(B_{z 0}\right)$ is the initial Hamiltonian and $\widetilde{\mathscr{H}}_{\text {Ising }}\left(B_{z \mathrm{c}}\right)$ is the final Hamiltonian, $s=t / T \in[0,1]$ is the normalized time, the function, $A(s)$, determines the adiabatic path with $A(0)=0$ and $A(1)=1$.

The time required for the adiabatic path is determined by the adiabatic condition ${ }^{42}$. The simplest adiabatic path is the linear path, which corresponds to $A(s)=s$. In this case the evolution time is of the order $1 / \Delta_{\min }^{2}$ (see Supplementary Note 1 ) with $\Delta_{\min }$ as the minimal energy gap between the ground state and the first excited state $^{30}$. In our case the energy gap is

$\Delta(s)=2 \sqrt{2 B_{x}^{2}+\left[1-B_{z_{0}}+B_{z_{0}} A(s)-B_{z} A(s)\right]^{2}}$,

with $\Delta_{\min }=2 \sqrt{2} B_{x}$. For the linear path we thus have $T \propto \frac{1}{B_{x}^{2}}$. The $\mathrm{QFI}$, which is $F_{\mathrm{Q}}(|\widetilde{g}\rangle) \approx \frac{1}{2 B_{x}^{2}}$, then scales only linearly with $T$. More efficient adiabatic evolutions are required to go beyond the shot noise limit. One choice is the local adiabatic path, which adjusts the evolution speed according to the local energy gap as $\frac{d A(s)}{d s}=$ $c \Delta^{2}(s)$ with $c$ as a constant ${ }^{43}$. In this case the evolution time is of the order $\frac{1}{\Delta_{\min }} \log \frac{1}{\Delta_{\min }}$ and the precision can go beyond the shot noise limit (see Supplementary Note 1). Compared with the linear path with a constant speed, the local adiabatic path modifies the speed of the evolution according to the local energy gap. Intuitively, it distributes more time at places of smaller energy gap and evolves faster at places where the energy gap is large. 
This can speed up the evolution while satisfying the adiabatic condition at all places. Consequently, the scaling of QFI beyond the shot noise limit can be achieved.

In our experiment we further optimize the adiabatic path numerically. The optimization is achieved as follows: (1) first set a threshold on the fidelity, which is denoted as $P_{\mathrm{c}}$ (in our case $P_{\mathrm{c}}=$ 0.9999); (2) start from $A(0)=0$, let $A_{1}$ be the minimal value such that $\left|\left\langle g\left(A_{1}\right)\left|\mathrm{e}^{-\mathrm{i} \mathscr{H} \text { ad }\left(A_{1}\right) \tau}\right| g(0)\right\rangle\right| \leq P_{c}$, here $\tau$ is a fixed constant and $|g(A)\rangle$ is the ground state of $\mathscr{H}_{\text {ad }}(A)$; iteratively, we set $A_{i+1}$ as the minimal value such that $\left|\left\langle g\left(A_{i+1}\right)\left|\mathrm{e}^{-\mathrm{i} \mathscr{H}_{\text {ad }}\left(A_{i+1}\right) \tau}\right| g\left(A_{i}\right)\right\rangle\right| \leq P_{c}$; (3) If $A_{N} \geq 1$, then set $A_{N}=1$ and the procedure terminates. An adiabatic path is then obtained with $A\left(\frac{i}{N}\right)=A_{i}$. When $P_{\mathrm{c}}$ is chosen sufficiently close to 1 , the obtained path guarantees that the evolved state stays close to the ground state along the path and the time of this path is $\alpha 1 / B_{x}$ (see 'Methods' and Supplementary Note 1). This path is obtained from the fidelity directly, while the linear and the local paths are based on the energy gap which is related to the fidelity in an indirect way. We simulate the evolution of different adiabatic paths with the full Hamiltonian and it can be seen from Fig. 1 that the numerically obtained path shows a better performance.

To saturate the QCRB, we need to perform the optimal measurement, which is the projective measurement on the eigenvectors of the symmetric logarithmic derivative (SLD). The SLD, denoted as $L$, can be obtained from the equation $\frac{\partial \rho\left(B_{z}\right)}{\partial B_{z}}=\frac{1}{2}\left[\rho\left(B_{z}\right) L+L \rho\left(B_{z}\right)\right]^{2,36,37}$. When $\rho\left(B_{z}\right)=|\widetilde{g}\rangle\langle\widetilde{g}|$, we have $L=2\left(\left|\partial_{B_{z}} \widetilde{g}\right\rangle\langle\widetilde{g}|+| \widetilde{g}\rangle\left\langle\partial_{B_{z}} \widetilde{g}\right|\right)$, whose eigenvectors are given by

$$
\begin{aligned}
& \left|v_{\text {Opt }}^{1}(\theta)\right\rangle=\sqrt{\frac{1-\sin \theta}{2}}|11\rangle+\frac{\cos \theta}{\sqrt{2(1-\sin \theta)}} \frac{|01\rangle+|10\rangle}{\sqrt{2}}, \\
& \left|v_{\text {Opt }}^{2}(\theta)\right\rangle=-\sqrt{\frac{1+\sin \theta}{2}}|11\rangle+\frac{\cos \theta}{\sqrt{2(1+\sin \theta)}} \frac{|01\rangle+|10\rangle}{\sqrt{2}} .
\end{aligned}
$$

Here $\theta$ takes the same value as in Eq. (6). This optimal measurement depends on $B_{z}$, and in practice it can be implemented adaptively with the estimated value $B_{z}$ based on the previously accumulated measurement data $a^{40,41,44-46}$.

\section{Experimental implementation}

We implement the protocol on the Bruker Avance III $400 \mathrm{MHz}$ $(9.4 \mathrm{~T})$ spectrometer at the room temperature. The two nuclear spins, as shown in Fig. 2, are ${ }^{13} \mathrm{C}$ and ${ }^{1} \mathrm{H}$ in the ${ }^{13} \mathrm{C}$-labeled
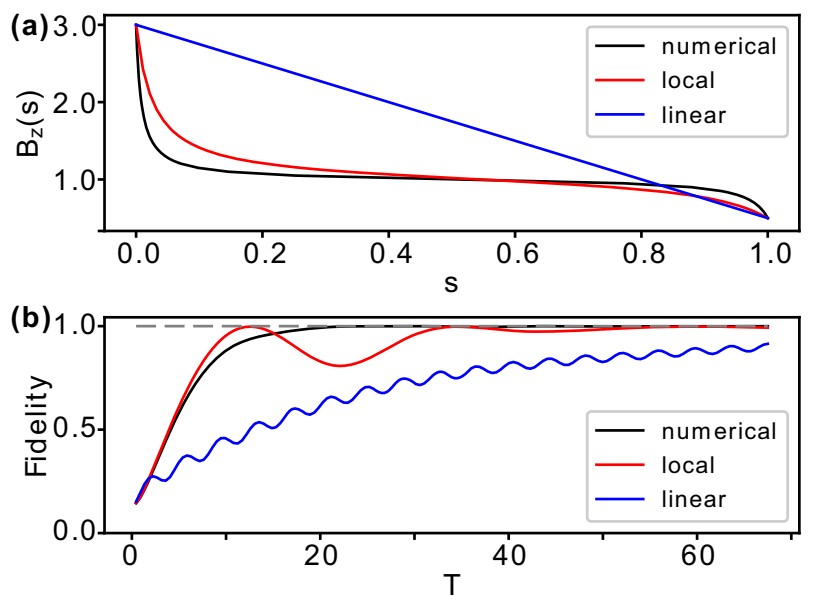

Fig. 1 Different adiabatic paths and the corresponding fidelity. a The linear adiabatic path, the local adiabatic path, and the numerically optimized path with $B_{x}=0.1$ and $B_{z}$ is adiabatically decreased from 3 to 0.5 . b Fidelity between the adiabatically evolved state and the actual ground state under these three paths when the total evolution time varies, here the unit is $2 /(\pi J)$. chloroform which is dissolved in $d_{6}$ acetone. In the doubleresonant rotating frame the natural Hamiltonian of this system is $\frac{\pi}{2} J \sigma_{z}^{1} \sigma_{z}^{2}$, where $J=214.5 \mathrm{~Hz}$ is the coupling strength. For convenience, we will take the time unit as $\frac{2}{\pi j}$ and write the Hamiltonian as $\mathscr{H}_{\mathrm{NMR}}=\sigma_{z}^{1} \sigma_{z}^{2}$. The transverse field can be realized by the onresonance radio-frequency pulse along the $x$-axis, and the vertical field can be generated with an appropriate offset of the transmitter's frequency ${ }^{47}$.

The initial state of the system is the pseudopure state (PPS), $\rho_{00}=\frac{1-\epsilon}{4} \mathbf{1}_{4}+\epsilon|00\rangle\left\langle\left. 00\right|^{48}\right.$, where $\epsilon \approx 10^{-5}$ represents the thermal polarization. We then prepare the ground state of the initial Hamiltonian $\mathscr{H}_{\text {ad }}[A(0)=0]=\widetilde{\mathscr{H}}_{\text {sing }}\left(B_{z 0}\right)$ and adiabatically drive the system to $\mathscr{H}_{\text {ad }}[A(T)=1]=\widetilde{\mathscr{H}}_{\text {Ising }}\left(B_{z f}\right)$, where $B_{z 0}$ is taken as 20 in the experiment and $B_{z f}=0$. In the experiment, we use the trotterized adiabatic evolution with $M$ segments ${ }^{49,50}$, each with a duration $\Delta t=T / M$. For the numerical path $T=c / B_{x}$ (in the experiment $c \approx 3.6$, see 'Methods' for the details) and the step number is taken as $M=100$ (see Supplementary Note 3 for the details). During each segment, the field is approximated as a constant with $B_{z}[i]=$ $\left[1-A\left(\frac{i}{M}\right)\right] B_{z 0}+A\left(\frac{i}{M}\right) B_{z f}$ and the corresponding evolution, as shown in Fig. $2 \mathrm{~b}$, is generated via the trotterization as $U_{i}(\Delta t)=$ $\mathrm{e}^{-\mathrm{i} \mathscr{H}_{\mathrm{ad}}\left[A_{i}\right] \Delta t}=\mathrm{e}^{-\mathrm{i} B_{x}\left(\sigma_{x}^{1}+\sigma_{x}^{2}\right) \frac{\Delta t}{2}} \mathrm{e}^{-\mathrm{i}\left\{B_{z}[i]\left(\sigma_{z}^{1}+\sigma_{z}^{2}\right)+\sigma_{z}^{1} \sigma_{z}^{2}\right\} \Delta t} \mathrm{e}^{-\mathrm{i} B_{x}\left(\sigma_{x}^{1}+\sigma_{x}^{2}\right) \frac{\Delta t}{2}}+O\left(\Delta t^{3}\right)$, where $\mathrm{e}^{-\mathrm{i} B_{x}\left(\sigma_{x}^{1}+\sigma_{x}^{2}\right) \frac{\Delta t}{2}}$ is realized by a strong resonant control pulse along the $x$-axis, $\mathrm{e}^{-\mathrm{i}\left[B_{z}[i]\left(\sigma_{z}^{1}+\sigma_{z}^{2}\right)+\sigma_{z}^{1} \sigma_{z}^{2}\right] \Delta t}$ is realized by a free evolution with an frequency offset $B_{z}[i] J / 2^{33}$.

In the experiment, we stop the adiabatic evolution at different $B_{z}$, which varies from 0.1 to 2.7 , to get the ground state $\left|\widetilde{g}^{\exp }\left(B_{z}\right)\right\rangle$, then perform the optimal projective measurements, $\left\{\left|v_{\text {Opt }}^{1}\left(B_{z}\right)\right\rangle\left\langle v_{\text {Opt }}^{1}\left(B_{z}\right)|,| v_{\text {Opt }}^{2}\left(B_{z}\right)\right\rangle\left\langle v_{\text {Opt }}^{2}\left(B_{z}\right)\right|\right\}$. In the experiment, only the local observables can be directly implemented. Specifically, the local observable implemented directly in our experiment is $\sigma_{x}^{1} \otimes \frac{1}{2}\left(\mathbf{1}_{2}-\sigma_{z}^{2}\right)$, whose eigenvectors are $\left|v_{\text {loc }}^{1}\right\rangle=$ $\frac{1}{\sqrt{2}}(|0\rangle+|1\rangle) \otimes|1\rangle$ and $\left|v_{\text {loc }}^{2}\right\rangle=\frac{1}{\sqrt{2}}(|0\rangle-|1\rangle) \otimes|1\rangle$ with the corresponding eigenvalues $\lambda_{1}=1$ and $\lambda_{2}=-1$. To perform the optimal measurement, we first implement a unitary operation $U_{\mathrm{O}}\left(B_{z}\right)$ with $U_{\mathrm{O}}\left(B_{z}\right)\left|v_{\text {Opt }}^{m}\right\rangle=\left|v_{\text {loc }}^{m}\right\rangle, m=1,2$, then perform the local measurement. The detailed implementation of $U_{\mathrm{O}}\left(B_{z}\right)$ can be found in the 'Methods' and Supplementary Note 4. In NMR the experimental signal corresponds to the average of the observable over an ensemble, which is given by $p_{1}\left(B_{z}\right) \lambda_{1}-p_{2}\left(B_{z}\right) \lambda_{2}$ with $p_{m}\left(B_{z}\right)=\left|\left\langle v_{\text {Opt }}^{m} \mid \widetilde{g}^{\exp }\left(B_{z}\right)\right\rangle\right|^{2}=\left|\left\langle v_{\text {loc }}^{m}\left|U_{O}\left(B_{z}\right)\right| \tilde{g}^{\exp }\left(B_{z}\right)\right\rangle\right|^{2}$. From the experimental signal, together with the condition $p_{1}\left(B_{z}\right)+p_{2}\left(B_{z}\right)$ $=1$, we can get $p_{1}\left(B_{z}\right)$ and $p_{2}\left(B_{z}\right)$, respectively. To get the Fisher information, $F_{C}^{\mathrm{Opt}}\left(B_{z}\right)=\frac{\left.\partial_{B_{z}} p_{1}\left(B_{z}\right)\right]^{2}}{p_{1}\left(B_{z}\right)}+\frac{\left[\partial_{B_{z}} p_{2}\left(B_{z}\right)\right]^{2}}{p_{2}\left(B_{z}\right)}$ (here $F_{C}$ is the classical Fisher information which equals to the QFI under the optimal measurement $)^{2}$, we also need to get $\partial_{B_{z}} p_{m}\left(B_{z}\right)$ experimentally. This is achieved by the difference method, i.e., by repeating the experiment at two neighboring points, $B_{z} \pm \delta$, where $\delta$ is a small shift (taken as 0.03 experimentally, see Supplementary Note 5 for details). The differentiation is then obtained as $\partial_{B_{z}} p_{m}\left(B_{z}\right) \approx \frac{p_{m}\left(B_{z}+\delta\right)-p_{m}\left(B_{z}-\delta\right)}{2 \delta}$.

The experiment is repeated under $B_{x}=0.1,0.2$, and 0.3 , where for each $B_{x}, B_{z}$ is varied non-uniformly from 0.1 to 2.7. As shown in Fig. 3a, under all $B_{x}$, the QFI around the critical point is significantly higher than the QFI away from the critical point. The total relative deviation of the experimental data from the numerical simulations is about $8.8 \%$ (see Supplementary Note 5). To show the practical advantage, we also plot the QFI per unit of time, $F_{\mathrm{Q}}(T) / T$, in Fig. 3b, which is also significantly higher around the critical point. This shows the critical point indeed provides an advantage in quantum metrology.

To demonstrate the scaling of the QFI with respect to the time, we perform another set of experiments where we adiabatically evolve the system from $B_{z 0}=20$ to the critical point, $B_{z c}=1$, with 
(a)

\begin{tabular}{|c|c|c|c|c|c|}
\hline & & ${ }^{13} C$ & ${ }^{1} H$ & $\mathrm{~T}_{1}$ & $\mathrm{~T}_{2}$ \\
\hline & ${ }^{13} C$ & $100 \mathrm{MHz}$ & & $18.5 \mathrm{~s}$ & $0.3 \mathrm{~s}$ \\
\hline & ${ }^{1} H$ & $214.6 \mathrm{~Hz}$ & $400 \mathrm{MHz}$ & $9.9 \mathrm{~s}$ & $3.3 \mathrm{~s}$ \\
\hline
\end{tabular}

(b)

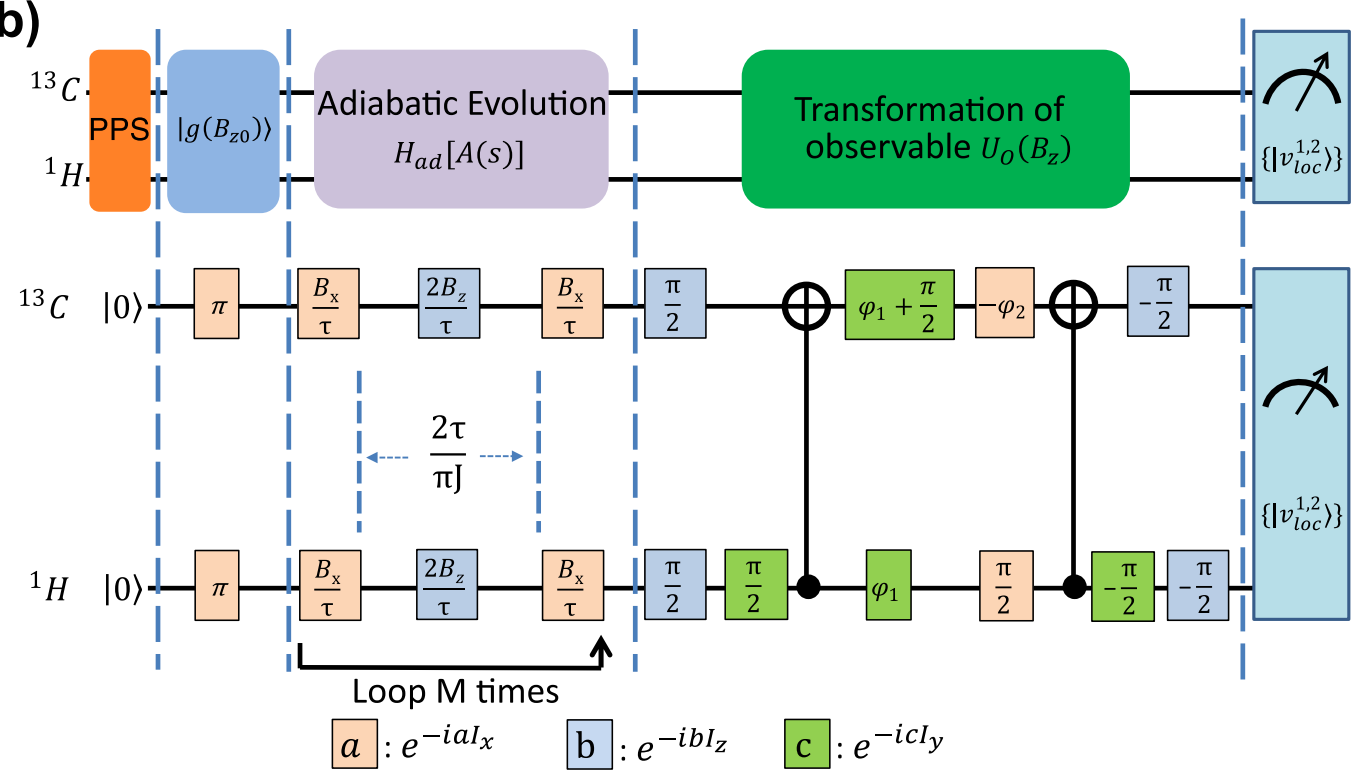

Fig. 2 Physical system and quantum circuit. a Molecular structure and relevant parameters of ${ }^{13} \mathrm{C}$-labeled chloroform. $\mathbf{b}$ Experimental scheme and quantum circuit for the critical quantum metrology with adiabatic evolution on NMR. Here, $\varphi_{1}=\arctan \left[\left(B_{z}-1\right) / 0\right]-\pi / 4, \varphi_{2}=|\theta-\pi / 2|$, where $\theta=\sqrt{2} B_{x} /\left(1-B_{z}\right)$. The specific pulse sequence for implementing in experiment can be seen in 'Methods' and Supplementary Note 4 .

$B_{x}$ tuned at different values to control the evolution time (since $T \propto 1 / B_{x}$ for the numerical adiabatic path). By experimentally obtaining the QFI under different $B_{x}$, we plot the relation of the QFI with the evolution time. As it can be seen from Fig. 3c, the QFI scales quadratically with the time. The total relative deviation of the experimental result from the numerical simulation is about $5.1 \%$ (see Supplementary Note 5). To better illustrate the scaling, we also plot $\sqrt{F_{\mathrm{Q}}}$ with respect to the time in Fig. $3 \mathrm{~d}$, where $\sqrt{F_{\mathrm{Q}}} \propto$ $T$ can be clearly seen. The coefficient of determination ${ }^{51}$ of the linear fitting is $98.6 \%$, and the slope of the fitted line is 0.31 with an uncertainty of 0.0032 . This clearly shows that the adiabatic scheme achieves the Heisenberg scaling near the critical point. We also numerically compare our protocol with the standard scheme of quantum metrology at the presence of noises (see Supplementary Note 7), and show it can surpass the standard scheme due to its robustness against decays.

\section{DISCUSSION}

Our work reports a joint theoretical and experimental study of critical quantum metrology by employing a first-order QPT in a minimal two-spin system. By introducing a small transverse magnetic field, we can not only encode the unknown parameter in the ground state but also tune the energy gap to control the evolution time of the local adiabatic passage which relieves the critical slowing down. With a numerically optimized path, we have implemented an adiabatic protocol to approach the critical point, where the precision achieves the Heisenberg scaling as $1 / T$, i.e., QFI scales as $T^{2}$. Due to the inherent robustness against decays of the adiabatic protocol ${ }^{29}$, the coherent evolution time is prolonged and the Heisenberg scaling can still be achieved near the critical point in a noisy environment. In contrast, in the standard scheme the Heisenberg scaling is not achievable in general for systems subject to noise, which can be recovered with quantum error correction $^{15}$. However, this is practically more challenging than our adiabatic scheme. It's worthy to mention that the first-order QPT in our employed model can occurs in a small quantum system and the energy gap near the critical point opens linearly with the small transverse field, which are promising properties for critical quantum metrology. Of course, increasing the quantum system size $N$ would bring more possibilities but greater challenges for the better performance of quantum metrology over classical methods. Due to the advantages of easy implementation, inherent robustness against decays and tunable energy gap, our adiabatic scheme is promising for exploring potential applications of critical quantum metrology on various physical systems, such as NV centers ${ }^{52}$, cold atoms $s^{53}$, and superconducting circuits ${ }^{54}$.

As a proof of principle, we assume a prior knowledge of the unknown estimated parameter in our critical quantum metrology protocol here. With little or no knowledge of the unknown estimated parameter, we can use a two-step adaptive method $^{4,41}$ in our present experimental setting to achieve the optimal measurement (saturating the QCRB), i.e., an initial static stage, and a second fully adaptive sequential stage. The ground state can be adaptively driven to the vicinity of the critical point of the first-order QPT, starting with a rough estimation of the unknown parameter from the static stage. Essentially, the adiabatic speed can be tuned based on the estimated value of the parameter with a slightly large constant to accommodate the possible difference between the estimated value and the true value (see Supplementary Note 2 for the details). 

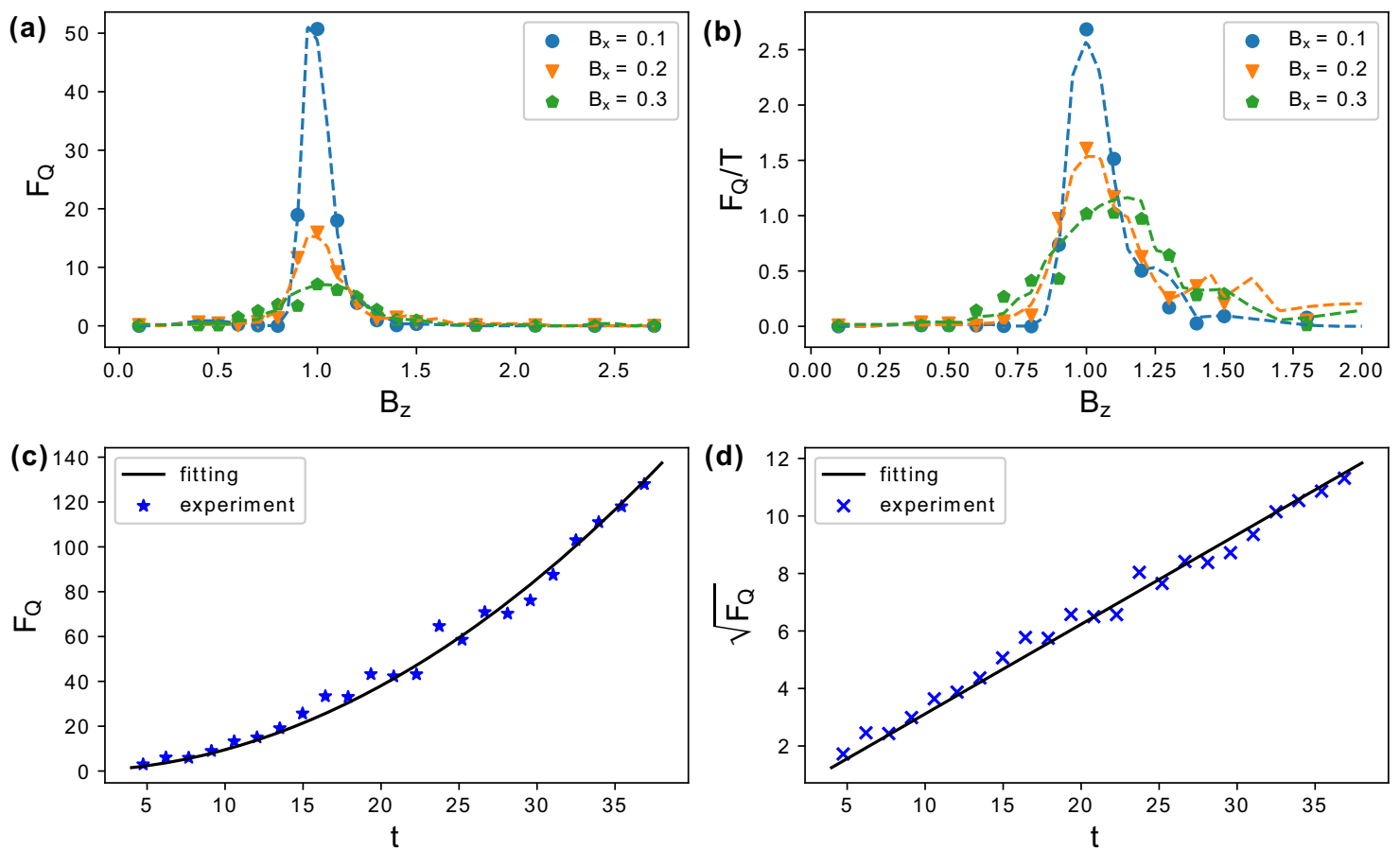

Fig. 3 Experimental measured QFI of adiabatically generated ground state. a Experimentally obtained QFI at different $B_{z}$ with $B_{x}=0.1,0.2$, and 0.3 (denoted by $\cdot \nabla, \triangle$, respectively), along with the corresponding numerical simulations (denoted by dashed lines) together for comparation. b The obtained QFI per unit of time at different $B_{z}$ with $B_{x}=0.1,0.2$, and 0.3 . c Experimentally obtained QFI (denoted by $\star$ ), and d its square root (denoted by $x$ ) near the critical point with different adiabatic time $t$, which is achieved by tuning $B_{x}$. The solid lines in (c) and (d) represent the fittings with a quadratic function and a linear function, respectively.

The adiabatic quantum metrology also connects the precision limit to the speed of the adiabatic evolution, various bounds in quantum metrology thus can also be used to study the speed limit of the adiabatic passage under noisy evolutions, which is another interesting direction to pursue. Evolutions that are beyond the adiabatic approximation, such as shortcuts to adiabaticity ${ }^{55}$, quench dynamics ${ }^{28}$, can also be investigated for further improvement of the critical metrology.

\section{METHODS}

\section{Numerical optimization of adiabatic paths and experimental implementation}

For the adiabatic path, $\mathscr{H}_{\text {ad }}[A(s)]=[1-A(s)] \mathscr{H}_{i}+A(s) \mathscr{H}_{f}$, the numerical path is obtained through the following steps:

- 1 : Set the step size for the change of $A(s)$ as $\Delta A \approx 0.001$, the step size of the evolution time as $\Delta t$, and a threshold for the fidelity as $P_{c}$, which is taken as 0.9999 in our case.

- 2: Start from the ground state of $\mathscr{H}_{i},|g(0)\rangle$, increase $A(s)$ by $\Delta A$ and evolve the state under the Hamiltonian for $\Delta t$ units of time, which leads to a state $\left|\phi_{0}(\Delta A)\right\rangle=\mathrm{e}^{-\mathrm{i} \mathscr{H}_{\text {ad }}(\Delta A) \Delta t}|g(0)\rangle$. Compute the fidelity between the state and the instantaneous ground-state $|g(\Delta A)\rangle$, which is $P_{\mathrm{t}}=\left|\left\langle g(\Delta A) \mid \phi_{0}(\Delta A)\right\rangle\right|$. If $P_{\mathrm{t}} \geq P_{\mathrm{c}}$ continue to increase $A(s)$ by $\Delta A$ until $P_{\mathrm{t}}=\left|\left\langle g\left[\left(n_{1}+1\right) \Delta A\right]\left|\mathrm{e}^{-\mathrm{i} \mathscr{H} \text { ad }\left[\left(n_{1}+1\right) \Delta A\right] \Delta t}\right| g(0)\right\rangle\right|<P_{\mathrm{c}}$. Set $A_{1}=n_{1} \Delta A$.

- 3: Similarly, start from the ground state of $\mathscr{H}_{\text {ad }}\left(A_{1}\right)$, and increase $A(s)$ till $P_{\mathrm{t}}=\left|\left\langle g\left[A_{1}+\left(n_{2}+1\right) \Delta A\right]\left|\mathrm{e}^{-\mathrm{i} \mathscr{H} \text { ad }\left[A_{1}+\left(n_{2}+1\right) \Delta A\right] \Delta t}\right| g\left(A_{1}\right)\right\rangle\right|<P_{\mathrm{c}}$. Set $A_{2}=$ $A_{1}+n_{2} \Delta A=n_{1} \Delta A+n_{2} \Delta A$. Similarly, we can get $A_{3}, A_{4}, \cdots, A_{n}, \cdots$.

- 4: When $A_{N}=\sum_{i=1,2, \ldots, N} n_{i} \Delta A \geq 1$, set $A_{N}=1$.

It can be proved that the time required for the numerical optimized path is in the order of $\frac{1}{B_{x}}$, which attains the Heisenberg scaling with $F_{\mathrm{Q}} \sim T^{2}$ (see Supplementary Note 1 for details).

It is difficult to experimentally realize so many segments in the numerical adiabatic path optimized above, consequently, we use the linear interpolation to construct $M+1$ segments for the experimental implementation. Here the adiabatic evolution was realized with $M+1$ discrete steps, in the ith step the evolution is governed by the Hamiltonian $\mathscr{H}_{\text {ad }}(A[i])$ with $A[i]=A\left(\frac{i}{M}\right)$, which corresponds to a constant field, $B_{z}[i]=\left[1-A\left(\frac{i}{M}\right)\right] B_{z 0}+A\left(\frac{i}{M}\right) B_{z f}$.

The evolution of each segment $U_{i}(\Delta t)=\mathrm{e}^{-\mathrm{i}\left[B_{z}[i]\left(\sigma_{z}^{1}+\sigma_{z}^{2}\right)+B_{x}\left(\sigma_{x}^{1}+\sigma_{x}^{2}\right)+\sigma_{z}^{1} \sigma_{z}^{2}\right] \Delta t}$, here $\Delta t=T /(M+1)$, is implemented approximately as $U_{i}^{\exp }(\Delta t)=$ $\mathrm{e}^{-\mathrm{i} B_{x}\left(\sigma_{x}^{1}+\sigma_{x}^{2}\right) \Delta t / 2} \mathrm{e}^{-\mathrm{i}\left[B_{z}[i]\left(\sigma_{z}^{1}+\sigma_{z}^{2}\right)+\sigma_{z}^{1} \sigma_{z}^{2}\right] \Delta t} \mathrm{e}^{-\mathrm{i} B_{x}\left(\sigma_{x}^{1}+\sigma_{x}^{2}\right) \Delta t / 2}$. Here $\Delta t$ and $M$ need to be optimized to satisfy: (i) $\Delta t$ is sufficient small so that $U_{i}(\Delta t)$ and $U_{i}^{\exp }(\Delta t)$ is sufficiently close for all $B_{z}[i]$; (ii) $\Delta t$ is not too small so that the number of the total segments, $M+1$, is not too big; and (iii) the total time $(M+1) \Delta t$ is not too large at the presence of the decoherence. We include the relaxation effect in the optimization, where for ${ }^{13} \mathrm{C}$ we take $T_{1}$ and $T_{2}$ as $18.5 \mathrm{~s}$ and $0.2 \mathrm{~s}$, respectively, and for ${ }^{1} \mathrm{H}$ we take $T_{1}$ and $T_{2}$ as $9.9 \mathrm{~s}$ and $0.6 \mathrm{~s}$, respectively.

For the case of $B_{x}=0.1$, upon the optimization $\Delta t$ is chosen as 0.36 (in the unit of $2 / \pi J)$ for which the fidelity between $U_{i}(\Delta t)$ and $U_{i}^{\exp }(\Delta t)$ is above $99.8 \%$ for all $B_{z}[i]$. We then increase the number of steps until it achieves the maximal average fidelity with $M+1=100$. The total adiabatic evolution time is then $T=M \Delta t=36$. Since for the numerical path we have $T \approx \frac{c}{B_{x^{\prime}}}$, then $c \approx T \times B_{x}=3.6$ in this case.

Similarly, for $B_{x}=0.2,0.3$, we take the same constant $c=3.6$ and the same number of segments (i.e., $M+1=100$ ) for consistency. The total adiabatic evolution time for $B_{x}=0.2$ is then $T=3.6 / 0.2=18$ and for $B_{x}=$ $0.3, T=3.6 / 0.3=12$.

\section{Experimental realization of optimal measurement}

In the experiment, the direct local observable is

$O_{\text {loc }}=\sigma_{x} \otimes|1\rangle\langle 1|$,

which can be diagonalized as

$O_{\text {loc }}=\sum_{1 \leq m \leq 4} \lambda_{m}\left|v_{\text {loc }}^{m}\right\rangle\left\langle v_{\text {loc }}^{m}\right|$,

here $\lambda_{1}=1, \lambda_{2}=-1, \lambda_{3}=\lambda_{4}=0,\left|v_{\text {loc }}^{1}\right\rangle=|+, 1\rangle,\left|v_{\text {loc }}^{2}\right\rangle=|-, 1\rangle,\left|v_{\text {loc }}^{3}\right\rangle=$ $|+, 0\rangle,\left|v_{\text {loc }}^{4}\right\rangle=|-, 0\rangle$ with $| \pm\rangle=\frac{|0\rangle \pm|1\rangle}{\sqrt{2}}$. 
The unitary operator that transforms the optimal observable to the local observable can be constructed as

$U_{\mathrm{O}}\left(B_{z}\right)=\sum_{m=1}^{4}\left|v_{\text {loc }}^{m}\right\rangle\left\langle v_{\mathrm{Opt}}^{m}\left(B_{z}\right)\right|$

where $\left|v_{\mathrm{Opt}}^{1}\left(B_{z}\right)\right\rangle$ and $\left|v_{\mathrm{Opt}}^{2}\left(B_{z}\right)\right\rangle$ are the basis of the optimal measurement given in the main text, and $\left|v_{\text {Opt }}^{3}\left(B_{z}\right)\right\rangle$ and $\left|v_{\text {Opt }}^{4}\left(B_{z}\right)\right\rangle$ are two additional vectors to form a complete orthonormal basis. Hence, the effective optimal observable we employed can be expressed as

$\mathrm{O}_{\mathrm{Opt}}\left(B_{z}\right)=\sum_{1 \leq m \leq 4} \lambda_{m}\left|v_{\mathrm{Opt}}^{m}\left(B_{z}\right)\right\rangle\left\langle v_{\mathrm{Opt}}^{m}\left(B_{z}\right)\right|$.

Without loss of generality, we can take

$\left|v_{\text {Opt }}^{1}\left(B_{z}\right)\right\rangle=\sqrt{\frac{1-\sin \theta}{2}}|11\rangle+\frac{\cos \theta}{\sqrt{2(1-\sin \theta)}} \frac{|01\rangle+|10\rangle}{\sqrt{2}}$,

$\left|v_{\text {Opt }}^{2}\left(B_{z}\right)\right\rangle=-\sqrt{\frac{1+\sin \theta}{2}}|11\rangle+\frac{\cos \theta}{\sqrt{2(1+\sin \theta)}} \frac{|01\rangle+|10\rangle}{\sqrt{2}}$,

$\left|v_{\text {Opt }}^{3}\left(B_{z}\right)\right\rangle=\sqrt{\frac{1-\sin \theta}{2}}|00\rangle+\frac{\cos \theta}{\sqrt{2(1-\sin \theta)}} \frac{|01\rangle-|10\rangle}{\sqrt{2}}$,

$\left|v_{\text {Opt }}^{4}\left(B_{z}\right)\right\rangle=-\sqrt{\frac{1+\sin \theta}{2}}|00\rangle+\frac{\cos \theta}{\sqrt{2(1+\sin \theta)}} \frac{|01\rangle-|10\rangle}{\sqrt{2}}$

with $\theta=\sqrt{2} B_{x} /\left(1-B_{z}\right)$.

It is easy to verify that $U_{\mathrm{O}}\left(B_{Z}\right) \in \mathbf{S O}(4)$, which can be decomposed as $U_{\mathrm{O}}\left(B_{z}\right)=\mathcal{M}(A \otimes B) \mathcal{M}^{\dagger 56}$ with $A, B \in \mathbf{S U}(2)$ and

$\mathcal{M}=\frac{1}{\sqrt{2}}\left(\begin{array}{cccc}1 & \mathrm{i} & 0 & 0 \\ 0 & 0 & \mathrm{i} & 1 \\ 0 & 0 & \mathrm{i} & -1 \\ 1 & -\mathrm{i} & 0 & 0\end{array}\right)$.

Any operator in $\mathbf{S U}(2)$ can be realized with three rotations via the Euler decomposition, we can thus write $A=R_{x}\left(a^{\mathrm{A}}\right) R_{y}\left(\beta^{\mathrm{A}}\right) R_{x}\left(\gamma^{\mathrm{A}}\right)$ and $B=R_{x}\left(a^{\mathrm{B}}\right)$ $R_{y}\left(\beta^{\mathrm{B}}\right) R_{x}\left(\gamma^{\mathrm{B}}\right)^{57}$. Then,

$U_{0}\left(B_{z}\right)=\mathcal{M}\left[R_{x}\left(a^{\mathrm{A}}\right) R_{y}\left(\beta^{\mathrm{A}}\right) R_{x}\left(\gamma^{\mathrm{A}}\right) \otimes R_{x}\left(a^{\mathrm{B}}\right) R_{y}\left(\beta^{\mathrm{B}}\right) R_{x}\left(\gamma^{\mathrm{B}}\right)\right] \mathcal{M}^{\dagger}$.

$\mathcal{M}$ can be decomposed as $\mathcal{M}=U_{\mathrm{CNOT}}\left[U_{\mathrm{S}} \otimes\left(U_{\mathrm{H}} U_{\mathrm{S}}\right)\right]$ with $U_{\mathrm{H}}=$ $\frac{1}{\sqrt{2}}\left(\begin{array}{cc}1 & 1 \\ 1 & -1\end{array}\right), U_{\mathrm{S}}=\frac{1}{\sqrt{2}}\left(\begin{array}{ll}1 & 0 \\ 0 & \mathrm{i}\end{array}\right)$ and $U_{\mathrm{CNOT}}$ as the CNOT gate,

$U_{\text {CNOT }}=\sqrt{\mathrm{i}} R_{z}^{1}(\pi / 2) R_{z}^{2}(-\pi / 2) R_{x}^{2}(\pi / 2) \mathrm{e}^{-\mathrm{i} \frac{\pi}{4} \sigma_{z}^{1} \sigma_{z}^{2}} R_{y}^{2}(\pi / 2)$,

here $R_{a}^{j}(\beta)=\mathrm{e}^{-\mathrm{i}_{2}^{\beta} \sigma_{\alpha}^{j}}$ denotes a rotation of the $j$ th spin around the axis $a \in$ $\{x, y, z\}$ with $\beta$-angle ${ }^{58}$.

\section{DATA AVAILABILITY}

The data that support the findings of this study are available from the corresponding authors upon reasonable request.

\section{CODE AVAILABILITY}

The codes for numerical simulation and data processing are available from the corresponding authors upon reasonable request.

Received: 21 May 2021; Accepted: 9 November 2021; Published online: 13 December 2021

\section{REFERENCES}

1. Holevo, A. S. Probabilistic and Statistical Aspects of Quantum Theory, Vol. 1 (Springer Science \& Business Media, 2011).

2. Helstrom, C. W. Quantum detection and estimation theory. J. Stat. Phys. 1, 231-252 (1969).

3. Cramér, H. Mathematical Methods of Statistics, Vol. 43 (Princeton University Press, 1999).

4. Giovannetti, V., Lloyd, S. \& Maccone, L. Advances in quantum metrology. Nat. Photonics 5, 222-229 (2011).

5. Giovannetti, V., Lloyd, S. \& Maccone, L. Quantum metrology. Phys. Rev. Lett. 96, 010401 (2006).
6. Giovannetti, V., Lloyd, S. \& Maccone, L. Quantum-enhanced measurements: beating the standard quantum limit. Science 306, 1330-6 (2004).

7. Escher, B. M., de Matos Filho, R. L. \& Davidovich, L. General framework for estimating the ultimate precision limit in noisy quantum-enhanced metrology. Nat. Phys. 7, 406-411 (2011).

8. Demkowicz-Dobrzanski, R., Kolodynski, J. \& Guta, M. The elusive Heisenberg limit in quantum-enhanced metrology. Nat. Commun. 3, 1063 (2012).

9. Yuan, H. \& Fung, C.-H. F. Quantum parameter estimation with general dynamics. npj Quantum Inf. 3, 14 (2017).

10. Wineland, D. J., Bollinger, J. J., Itano, W. M., Moore, F. L. \& Heinzen, D. J. Spin squeezing and reduced quantum noise in spectroscopy. Phys. Rev. A 46, R6797-R6800 (1992).

11. Pezzé, L. \& Smerzi, A. Entanglement, nonlinear dynamics, and the Heisenberg limit. Phys. Rev. Lett. 102, 100401 (2009).

12. Huang, J., Wu, S., Zhong, H. \& Lee, C. Quantum metrology with cold atoms. In Annual Review of Cold Atoms and Molecules (eds Madison, K., Bongs, K., Carr, L. D., Rey, A. N. \& Zhai, H.) 365-415 (World Scientific, 2014).

13. Huang, J., Qin, X., Zhong, H., Ke, Y. \& Lee, C. Quantum metrology with spin cat states under dissipation. Sci. Rep. 5, 17894 (2015).

14. Demkowicz-Dobrzanski, R. \& Maccone, L. Using entanglement against noise in quantum metrology. Phys. Rev. Lett. 113, 250801 (2014).

15. Zhou, S., Zhang, M., Preskill, J. \& Jiang, L. Achieving the Heisenberg limit in quantum metrology using quantum error correction. Nat. Commun. 9, 78 (2018).

16. Rams, M. M., Sierant, P., Dutta, O., Horodecki, P. \& Zakrzewski, J. At the limits of criticality-based quantum metrology: apparent super-Heisenberg scaling revisited. Phys. Rev. X 8, 021022 (2018).

17. Garbe, L., Bina, M., Keller, A., Paris, M. G. A. \& Felicetti, S. Critical quantum metrology with a finite-component quantum phase transition. Phys. Rev. Lett. 124, 120504 (2020).

18. Zanardi, P. \& Paunković, N. Ground state overlap and quantum phase transitions. Phys. Rev. E 74, 031123 (2006).

19. You, W.-L., Li, Y.-W. \& Gu, S.-J. Fidelity, dynamic structure factor, and susceptibility in critical phenomena. Phys. Rev. E 76, 022101 (2007).

20. Zanardi, P., Paris, M. G. A. \& Campos Venuti, L. Quantum criticality as a resource for quantum estimation. Phys. Rev. A 78, 042105 (2008).

21. Invernizzi, C., Korbman, M., Campos Venuti, L. \& Paris, M. G. A. Optimal quantum estimation in spin systems at criticality. Phys. Rev. A 78, 042106 (2008).

22. Salvatori, G., Mandarino, A. \& Paris, M. G. A. Quantum metrology in lipkinmeshkov-glick critical systems. Phys. Rev. A 90, 022111 (2014).

23. Bina, M., Amelio, I. \& Paris, M. G. A. Dicke coupling by feasible local measurements at the superradiant quantum phase transition. Phys. Rev. E 93, 052118 (2016).

24. Boyajian, W. L., Skotiniotis, M., Dür, W. \& Kraus, B. Compressed quantum metrology for the ising hamiltonian. Phys. Rev. A 94, 062326 (2016).

25. Mehboudi, M., Correa, L. A. \& Sanpera, A. Achieving sub-shot-noise sensing at finite temperatures. Phys. Rev. A 94, 042121 (2016).

26. Frérot, I. \& Roscilde, T. Quantum critical metrology. Phys. Rev. Lett. 121, 020402 (2018).

27. Ivanov, P. A. \& Porras, D. Adiabatic quantum metrology with strongly correlated quantum optical systems. Phys. Rev. A 88, 023803 (2013).

28. Chu, Y., Zhang, S., Yu, B. \& Cai, J. Dynamic framework for criticality-enhanced quantum sensing. Phys. Rev. Lett. 126, 010502 (2021).

29. Childs, A. M., Farhi, E. \& Preskill, J. Robustness of adiabatic quantum computation. Phys. Rev. A 65, 012322 (2001).

30. Farhi, E., Goldstone, J., Gutmann, S. \& Sipser, M. Quantum computation by adiabatic evolution. Preprint at https://arxiv.org/abs/quant-ph/0001106 (2000).

31. Aharonov, D. et al. Adiabatic quantum computation is equivalent to standard quantum computation. SIAM J. Comput. 37, 166-194 (2007).

32. Albash, T. \& Lidar, D. A. Adiabatic quantum computation. Rev. Mod. Phys. 90, 015002 (2018).

33. Peng, X., Du, J. \& Suter, D. Quantum phase transition of ground-state entanglement in a Heisenberg spin chain simulated in an nmr quantum computer. Phys. Rev. A 71, 012307 (2005).

34. Zhuang, M., Huang, J., Ke, Y. \& Lee, C. Quantum adiabatic evolution: symmetry protected quantum adiabatic evolution in spontaneous symmetry breaking transitions. Ann. Phys. 532, 2070020 (2020).

35. Ovchinnikov, A. A., Dmitriev, D. V., Krivnov, V. Y. \& Cheranovskii, V. O. Antiferromagnetic ising chain in a mixed transverse and longitudinal magnetic field. Phys. Rev. B 68, 214406 (2003).

36. Braunstein, S. L. \& Caves, C. M. Statistical distance and the geometry of quantum states. Phys. Rev. Lett. 72, 3439-3443 (1994).

37. Braunstein, S. L., Caves, C. M. \& Milburn, G. J. Generalized uncertainty relations: theory, examples, and lorentz invariance. Ann. Phys. (NY) 247, 135-173 (1996).

38. Zhang, J., Peng, X., Rajendran, N. \& Suter, D. Detection of quantum critical points by a probe qubit. Phys. Rev. Lett. 100, 100501 (2008). 
39. Yuan, H. \& Fung, C. H. Optimal feedback scheme and universal time scaling for hamiltonian parameter estimation. Phys. Rev. Lett. 115, 110401 (2015).

40. Fujiwara, A. Strong consistency and asymptotic efficiency for adaptive quantum estimation problems. J. Phys. A: Math. Gen. 39, 12489-12504 (2006).

41. GILL, R. D. Conciliation of Bayes and Pointwise Quantum State Estimation, 239-261 (World Scientific, 2008).

42. Jansen, S., Ruskai, M.-B. \& Seiler, R. Bounds for the adiabatic approximation with applications to quantum computation. J. Math. Phys. 48, 102111 (2007).

43. Roland, J. \& Cerf, N. J. Quantum search by local adiabatic evolution. Phys. Rev. A 65, 042308 (2002).

44. Nagaoka, H. An asymptotically efficient estimator for a one-dimensional parametric model of quantum statistical operators. In Proc. 1988 IEEE Int. Symposium on Information Theory, Vol. 198 (1988).

45. Nagaoka, H. On the parameter estimation problem for quantum statistical models. In Asymptotic Theory of Quantum Statistical Inference (ed. Hayashi, M.) 125-132 (World Scientific, 2005).

46. Hayashi, M. \& Matsumoto, K. Asymptotic performance of optimal state estimation in qubit system. J. Math. Phys. 49, 102101 (2008).

47. Jones, J. A. et al. Magnetic field sensing beyond the standard quantum limit using 10-spin noon states. Science 324, 1166-1168 (2009).

48. Peng, X. et al. Preparation of pseudo-pure states by line-selective pulses in nuclear magnetic resonance. Chem. Phys. Lett. 340, 509-516 (2001).

49. Sun, Y., Zhang, J.-Y., Byrd, M. S. \& Wu, L.-A. Trotterized adiabatic quantum simulation and its application to a simple all-optical system. New J. Phys. 22, 053012 (2020).

50. Wu, L.-A., Byrd, M. S. \& Lidar, D. A. Polynomial-time simulation of pairing models on a quantum computer. Phys. Rev. Lett. 89, 057904 (2002).

51. Devore, J. L. Probability and Statistics for Engineering and the Sciences 8th edn. (Cengage Learning, 2011).

52. Liu, G. Q. et al. Demonstration of entanglement-enhanced phase estimation in solid. Nat. Commun. 6, 6726 (2015).

53. Napolitano, M. et al. Interaction-based quantum metrology showing scaling beyond the Heisenberg limit. Nature 471, 486-489 (2011).

54. Braumuller, J. et al. Analog quantum simulation of the rabi model in the ultrastrong coupling regime. Nat. Commun. 8, 779 (2017).

55. del Campo, A. Shortcuts to adiabaticity by counterdiabatic driving. Phys. Rev. Lett 111, 100502 (2013)

56. Vatan, F. \& Williams, C. Optimal quantum circuits for general two-qubit gates. Phys. Rev. A 69, 032315 (2004).

57. Nielsen, M. A. \& Chuang, I. Quantum Computation and Quantum Information 239-261 (Cambridge University Press, 2010).

58. Gershenfeld, N. A. \& Chuang, I. L. Bulk spin-resonance quantum computation. Science 275, 350-356 (1997).

\section{ACKNOWLEDGEMENTS}

The authors thank Prof. Youjin Deng for useful discussions. This work is supported by National Key Research and Development Program of China (Grant No.
2018YFA0306600), the National Natural Science Foundation of China (Grant No. 11927811, Grant No. 12004371), Anhui Initiative in Quantum Information Technologies (Grant No. AHY050000), Research Grants Council of Hong Kong (GRF No. 14308019), and the Research Strategic Funding Scheme of The Chinese University of Hong Kong (No. 3133234).

\section{AUTHOR CONTRIBUTIONS}

X.P. and H.Y. conceived the project. H.Y. and Y.C. conceived the relevant theoretical constructs. X.P. and R.L designed the experiment. R.L. and Y.C. performed the measurements and analyzed the data. M.J. assisted with the experiment. All authors contributed to analyzing the data, discussing the results, and writing the paper.

\section{COMPETING INTERESTS}

The authors declare no competing interests.

\section{ADDITIONAL INFORMATION}

Supplementary information The online version contains supplementary material available at https://doi.org/10.1038/s41534-021-00507-x.

Correspondence and requests for materials should be addressed to Haidong Yuan or Xinhua Peng.

Reprints and permission information is available at http://www.nature.com/ reprints

Publisher's note Springer Nature remains neutral with regard to jurisdictional claims in published maps and institutional affiliations.

(i) Open Access This article is licensed under a Creative Commons Attribution 4.0 International License, which permits use, sharing, adaptation, distribution and reproduction in any medium or format, as long as you give appropriate credit to the original author(s) and the source, provide a link to the Creative Commons license, and indicate if changes were made. The images or other third party material in this article are included in the article's Creative Commons license, unless indicated otherwise in a credit line to the material. If material is not included in the article's Creative Commons license and your intended use is not permitted by statutory regulation or exceeds the permitted use, you will need to obtain permission directly from the copyright holder. To view a copy of this license, visit http://creativecommons. org/licenses/by/4.0/.

(c) The Author(s) 2021 